\title{
Italian experience with rVIII-single chain: a survey of patients with haemophilia A and their physicians
}

\author{
Alessandra Borchiellini ${ }^{1}{ }^{10} \cdot$ Giancarlo Castaman $^{2} \cdot$ Giulio Feola $^{3} \cdot$ Antonietta Ferretti $^{4} \cdot$ Paola Giordano $^{5}$. \\ Matteo Luciani ${ }^{6}$. Giuseppe Malcangi ${ }^{7} \cdot$ Maurizio Margaglione $^{8} \cdot$ Angelo Claudio Molinari $^{9} \cdot$ Berardino Pollio $^{10}$. \\ Angiola Rocino ${ }^{11} \cdot$ Cristina Santoro $^{12} \cdot$ Michele Schiavulli $^{13} \cdot$ Ezio Zanon $^{14}$
}

Accepted: 28 October 2021 / Published online: 13 November 2021

(c) The Author(s) 2021

\begin{abstract}
rVIII-SingleChain is indicated for treatment and prophylaxis of bleeding in patients with haemophilia A (HA). The safety and efficacy of rVIII-SingleChain have previously been shown in the AFFINITY clinical trial programme. This survey evaluated clinical experience following a switch to rVIII-SingleChain from the perspective of both physicians and patients. A web-based survey (July-September 2019) involving 14 Haemophilia Treatment Centres (HTCs) collected data about HA patients who were under treatment with rVIII-SingleChain for $\geq 12$ months, as reported by their physicians. In addition, about half of these patients were separately interviewed. Out of 91 patients receiving rVIII-SingleChain in the 14 participating HTCs, 48 had been treated for $\geq 12$ months; among those $48,38 \%$ were $\leq 18$ years, $37 \% 19-40$ years and $25 \% \geq 41$ years; $73 \%$ of them had severe HA and $85 \%$ were being treated with prophylactic therapy. Twenty-six patients accepted to be separately interviewed: mean age was 30 years; $62 \%$ had severe HA and $85 \%$ were receiving prophylaxis. Focusing on those patients who were already in prophylaxis with prior FVIII (all but one with recombinant factors), infusion frequency was significantly reduced from 3-2 per week following the switch to rVIII-SingleChain (mean, 2.74 vs. 2.44, respectively; $\mathrm{p}=0.013$ ), as reported by physicians; the rate of patients needing 3 infusions per week dropped from $74 \%$ with previous products to $44 \%$ with rFVIII-SingleChain. The annual mean factor consumption was $4740 \mathrm{IU} / \mathrm{Kg}$ (median, $4500 \mathrm{IU} / \mathrm{Kg}$; min, 2.215 IU/Kg; max, $7.200 \mathrm{IU} / \mathrm{Kg}$ ) with prior product and $4320 \mathrm{IU} / \mathrm{Kg}$ (median, $4320 \mathrm{IU} / \mathrm{Kg}$; min, $2.215 \mathrm{IU} / \mathrm{Kg}$; max, 6.646 $\mathrm{IU} / \mathrm{Kg}$ ) with rVIII-SingleChain. Both physicians and patients reported a significant reduction in annual total bleeding rates with rVIII-SingleChain compared with prior product (mean 2.15-0.96 and 2.46-0.71 events/year, $\mathrm{p}=0.031$ and $\mathrm{p}=0.018$, respectively). Mean satisfaction ratings (from 1; dissatisfied, to 5; very satisfied) for rVIII-SingleChain were quite high for both physicians $(4.14,86 \%$ satisfied/very satisfied) and patients $(4.18,86 \%$ satisfied/very satisfied). This survey suggested that switching to rVIII-SingleChain allowed patients to reduce their injection frequency without increasing factor consumption or compromising clinical results. Both physicians and patients reported a positive experience with rVIII-SingleChain after 1 year of treatment.
\end{abstract}

Keywords Haemophilia A · rVIII-SingleChain · Injection frequency

\section{Highlights}

- Clinicians indicated rVIII-SingleChain as a reliable choice with a favorable pharmacokinetic profile.

- Switching to rVIII-SingleChain allowed patients to reduce their injection frequency without increasing factorconsumption or compromising clinical results.

Alessandra Borchiellini

aborchiellini@cittadellasalute.to.it

Extended author information available on the last page of the article
- Both physicians and patients reported a positive experience with rVIII-SingleChain after 1 year of treatment.

- Results of this real-world study validate clinical trials' results in the daily clinical practice. 


\section{Introduction}

Haemophilia A (HA) is an X-linked congenital bleeding disorder resulting from mutations in the gene encoding the coagulation factor VIII (FVIII) [1]. Regular prophylactic factor VIII replacement is currently the standard treatment for patients with severe haemophilia A (defined as FVIII levels $<1 \mathrm{IU} / \mathrm{dL}$ ), and its introduction has greatly improved the prognosis, life expectancy, and quality of life (QoL) of subjects with haemophilia, particularly preventing spontaneous bleeding episodes into the joints or the muscles, and the developing of haemophilic arthropathy [2, 3]. However, issues related to the burden of treatment, due to the need for frequent injections, and to the serious complication of inhibitor development, still calls for the need of effective new agents with improved stability and reduced immunogenicity.

rVIII-SingleChain is a novel recombinant FVIII (rFVIII) molecule designed to provide improved stability compared with other available rFVIII, through the formation of a covalent linkage between the light and heavy chains of FVIII [4]. The resulting single-chain $\mathrm{rFVIII}$ molecule demonstrated enhanced PK parameters [5], and higher affinity for von Willebrand factor (VWF). VWF helps stabilizing FVIII and protects it from premature clearance, potentially also playing a role in reducing the development of inhibitors by limiting the recognition of rFVIII by antigen-presenting cells [4]. Thanks to its innovative features, rVIII-SingleChain allows for less frequent prophylaxis regimes (i.e. 2-3 times per week) compared to standard half-life (SHL) rFVIII products, which typically require 3-4 infusions per week, with major advantages in terms of burden of care and compliance to treatment for patients [6]. Since even most effective current prophylactic regimens may not completely prevent joint disease, in a long-term perspective adherence to therapy has important implications for the functional outcome of haemophilic patients.

The efficacy and safety of rVIII-SingleChain in previously treated adult/adolescent and children patients with severe HA were evaluated in the AFFINITY series of clinical trials, involving a total of 259 patients including 84 children [7, 8]. RVIII-SingleChain when administered on-demand or in a prophylaxis regimen demonstrated to effectively control bleeding episodes, with high treatment success rates and good/excellent haemostatic efficacy in more than $90 \%$ of cases, and to be associated with low annualized bleeding rates in patients on prophylaxis. Surgical haemostasis with rVIII-SingleChain was also effective during major surgeries in adult and adolescent patients and rated as excellent in $94 \%$ of surgeries [7]. None of the participant to the trials developed FVIII inhibitors [7, 8].

A key point in the clinical use of rVIII-SingleChain is the dosing regimen in patients receiving routine prophylaxis.
Confirming the benefits of rVIII-SingleChain improved stability, a consistent proportion of adult or children patients enrolled in the two pivotal trials were able to reduce their injection frequency compared with their pre-study regimen $[7,8]$. These findings were recently confirmed by real-world studies performed in Europe (Germany) and United States, which reported reduced dosing frequency and consumption compared with prior treatment in patients with haemophilia A who switched to rVIII-SingleChain prophylaxis, with similar bleeding rates $[6,9]$.

In the present study, we report a national survey among Italian physicians and patients treated for at least 12 months with rVIII-SingleChain, with the aim of obtaining a realworld picture of doctors' and patients' experiences and perceptions about the drug and collecting data on the number of injections, consumption of FVIII, and number of bleeding episodes in patients receiving rVIII-SingleChain. The results presented in this paper offer a quite comprehensive picture of rVIII-SingleChain good clinical results in real life.

\section{Methods}

A web-based survey was designed to collect data about HA patients who were under treatment with rVIII-SingleChain for $\geq 12$ months, as reported by their physicians. In addition, part of these patients were separately interviewed. The survey, involving 14 Italian Haemophilia Treatment Centres (HTCs), was carried out between July and September 2019, with the support of IQVIA ${ }^{\mathrm{TM}}$, who also performed all data analyses.

\section{Survey design}

The data collection included a physicians' phase and a patients' phase. For the physicians' phase, 14 specialists from 14 HCTs were questioned by computer-assisted web interviewing (CAWI) interviews lasting $30 \mathrm{~min}$. Patients treated with rVIII-SingleChain for at least 12 months could be included in the analysis. The questionnaire comprised two sections: a general section, reporting a comprehensive description of physician's experience with HA patients receiving rVIII-SingleChain (cohort A:48 patients), and a section comprising detailed records of single patients treated with rVIII-SingleChain, when available (cohort B: 38 patients, one case record for each patient). For the patients' phase, physicians sent a web link to their patients receiving rVIII-SingleChain for $\geq 12$ months, 26 of whom anonymously completed an on-line questionnaire about their experience with rVIII-SingleChain (cohort C:26 patients). The on-line questionnaires for doctors and patients were designed to allow for a comparison between the clinician's 
Fig. 1 Survey design and patients' disposition
Interview-based data collection (Jul-Sep 2019)

14 clinicians from 14 I Italian HTCs

$\sqrt{8}$

91 HA patients without inhibitors and being treated with rVIII-SingleChain

$48 \mathrm{HA}$ patients receiving $\mathrm{VVIII-SingleChain} \mathrm{for} \geq 1$ year General patients' population description by physicians

$\sqrt{2}$

\section{HA patients described in detail by physicians \\ Single patient case records}

26 HA patients described in detail by the patients themselves

Single patient case records
General analysis

Detailed analysis (physician's and patient's point of view) point of view and the patient's direct experience, mostly including analogous questions. However, the population of patients interviewed did not necessarily correspond to that described by the physicians (Fig. 1).

\section{Data collected}

The main outcomes assessed by the survey were: description of patients treated with rVIII-SingleChain for at least 12 months (general description of cohort A patients and detailed description of cohort B patients by clinicians from clinical records; detailed description of cohort $\mathrm{C}$ using records compiled by the patients themselves), including treatment prior to prophylaxis with rVIII-SingleChain and pharmacokinetic assessment; changes observed after 12 months of therapy with rVIII-SingleChain in terms of dosage regimen, FVIII consumption, and rate of annual total bleeds (ABR) and joint bleeds (AJBR); level and areas of satisfaction with rVIII-SingleChain by the physicians' and patients' perspective (satisfaction ratings, from 1 , dissatisfied; to 5 , very satisfied); patient's general experience with the disease and the prophylactic therapy, relationship with the Haemophilia Centre, level of information on haemophilia.

\section{Statistical analysis}

Standard descriptive statistics were used to analyse the survey results. Categorical data were described by numbers and percentages, and continuous variables by mean or median and ranges. To assess differences in outcome before and after the switch to rVIII-SingleChain, the Student $t$ test, specifically indicated for small sample sizes ( $<30$ cases), was employed. A P value $<0.05$ was considered statistically
Table 1 Demographic and clinical characteristics of the total population of patients (cohort A)

\begin{tabular}{ll}
\hline Characteristics & Number $(\%)$ \\
\hline Number of patients & 48 \\
Age & \\
$0-12$ years & $10(21)$ \\
$13-18$ years & $8(17)$ \\
$19-40$ years & $18(37)$ \\
$41-65$ years & $9(19)$ \\
$>65$ years & $3(6)$ \\
Haemophilia severity & \\
Mild & $3(6)$ \\
Moderate & $10(21)$ \\
Severe & $35(73)$ \\
Treatment regimen & \\
On-demand & $7(15)$ \\
Prophylaxis & $41(85)$ \\
Previous treatment (prophylaxis pts. n=41) & \\
PUPs & $1(2)$ \\
PTPs on-demand & $5(12)$ \\
PTPs on regular prophylaxis & $35(86)$ \\
\hline
\end{tabular}

PUPs previously untreated patients, $P T P s$ previously treated patients

significant. All statistical analyses were performed by IQVIA $^{\mathrm{TM}}$.

\section{Results}

\section{General description of patients' population}

A total of 14 physicians from 14 Italian HTCs were enrolled in the study. Overall, they reported a total of 91 HA patients 
Table 2 Demographic and clinical characteristics of patients analysed in detail by clinicians' (cohort B) and patients' (cohort C)

\begin{tabular}{|c|c|c|c|c|}
\hline Characteristics & \multicolumn{2}{|c|}{ Clinicians' point of view (cohort B) } & \multicolumn{2}{|c|}{$\begin{array}{l}\text { Patients' point of view (cohort } \\
\text { C) }\end{array}$} \\
\hline \multicolumn{5}{|l|}{ Total } \\
\hline Number of patients & \multicolumn{2}{|l|}{38} & \multicolumn{2}{|l|}{26} \\
\hline Age, mean & \multicolumn{2}{|l|}{33 years } & \multicolumn{2}{|l|}{30 years } \\
\hline \multicolumn{5}{|l|}{ Haemophilia severity } \\
\hline Mild (\%) & \multicolumn{2}{|l|}{$3(8)$} & \multicolumn{2}{|l|}{$4(15)$} \\
\hline Moderate (\%) & \multicolumn{2}{|l|}{$9(24)$} & \multicolumn{2}{|l|}{$6(23)$} \\
\hline Severe $(\%)$ & \multicolumn{2}{|l|}{$26(68)$} & \multicolumn{2}{|l|}{$16(62)$} \\
\hline Treatment duration, mean & \multicolumn{2}{|l|}{14.6 months } & \multicolumn{2}{|l|}{15.1 months } \\
\hline By treatment regimen & On-demand & Prophylaxis & On-demand & Prophylaxis \\
\hline Number of patients (\%) & $6(16)$ & $32(84)$ & $4(15)$ & $22(85)$ \\
\hline Age, mean & 38 years & 32 years & 44 years & 27 years \\
\hline Weight, mean & $71 \mathrm{~kg}$ & $63 \mathrm{~kg}$ & $82 \mathrm{~kg}$ & $61 \mathrm{~kg}$ \\
\hline \multicolumn{5}{|l|}{ Disease severity } \\
\hline Mild (\%) & $3(50)$ & 0 & $2(50)$ & $2(9)$ \\
\hline Moderate (\%) & $1(17)$ & $8(25)$ & $1(25)$ & $5(23)$ \\
\hline Severe $(\%)$ & $2(33)$ & $24(75)$ & $1(25)$ & $15(68)$ \\
\hline \multicolumn{5}{|l|}{ Sport practice* } \\
\hline Yes $(\%)$ & $2(33)$ & $14(44)$ & $2(50)$ & $13(59)$ \\
\hline No $(\%)$ & $4(67)$ & $18(56)$ & $2(50)$ & $9(41)$ \\
\hline
\end{tabular}

*Sports done: Swimming, gym, competitive football, non-competitive football, horse-riding, tennis, beach volleyball, weights, kickboxing, basketball, running, martial arts without inhibitors being treated with rVIII-SingleChain under the Centre's care. Out of these, 48 patients (53\%) had been receiving the drug for at least 12 months (mean followup: 15.8 months), and were included in the general population (cohort A). Demographic and clinical characteristics of all enrolled patients are summarized in Table 1.

Out of these 48 patients, $38 \%$ were $\leq 18$ years, $37 \%$ $19-40$ years and $25 \geq 41$ years. Forty-one/48 patients $(85 \%)$ were on a regular prophylaxis regimen with rVIII-SingleChain; of these, 33 (80\%) had a severe form of haemophilia and $8(20 \%)$ a moderate form. The majority of patients on prophylaxis $(n=35,86 \%)$ were already treated with a prophylactic regimen before the switch to rVIII-SingleChain; $5(12 \%)$ received an on-demand therapy and 1 patient $(2 \%)$ was previously untreated (PUP) (Table 1). The most frequent previous medication used for prophylaxis by the 35 patients was a full length 2 nd generation rFVIII from baby hamster kidney (BHK) cells (Helixate NG) (66); 17\% were treated with 3rd generation rFVIII; $14 \%$ with other 1 st/2nd generation rFVIII, and one patients (3\%) with a plasma-derived FVIII product (3\%). No one used extended half life (EHL) products

\section{PK monitoring}

Among the total population of 48 patients treated with rVIIISingleChain for at least 1 year (cohort A), 56\% of cases (27 patients) underwent pharmacokinetic (PK) studies in the last 12 months during their transition to the new drug or later to optimize their rVIII-SingleChain treatment. $21 \%$ of Centres used the one-stage clotting assay for plasma FVIII activity monitoring, $7 \%$ the chromogenic test and $72 \%$ used both methods. Since the one-stage clotting assay underestimates rVIII-SingleChain by about $50 \%$, the result must be multiplied by a factor of two to determine the patient's FVIII activity level (as indicated in the product's SPC) 4. The use of the conversion factor for rVIII-SingleChain was identified as a drawback by $23 \%$ of interviewed physicians, because of poor sensitivity to low values, occasional unexpected data or potential errors by clinicians or laboratory technicians not expert in haemophilia.

\section{Detailed records of patients treated with rVIII-SingleChain}

To gather more in-depth information about clinical results and satisfaction with the current treatment, detailed case records of patients being treated with rVIII-SingleChain for at least 12 months were obtained by clinicians (cohort $\mathrm{B}, \mathrm{n}=38$ ) and by the patients themselves (cohort $\mathrm{C}, \mathrm{n}=26$ ). Demographic and clinical data of these subgroups of patients are reported in Table 2.

On the whole, patients receiving rVIII-SingleChain were young (mean age of 30-33 years) and regularly practicing 
Fig. 2 Number of weekly infusions before and after treatment with rVIII-SingleChain
Clinicians' point of view ( $n=27)$

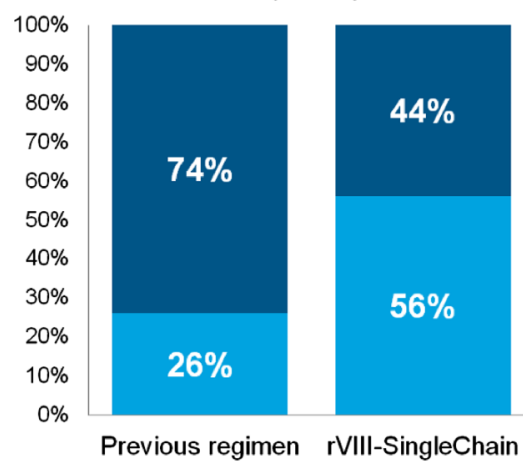

Patients' point of view

$(n=20)$

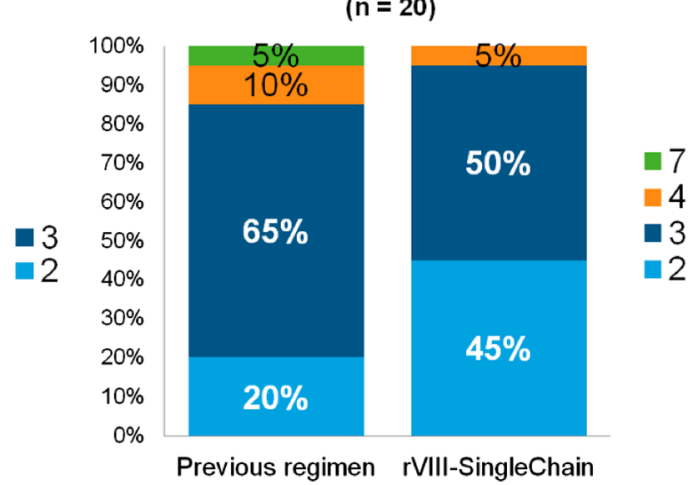

\begin{tabular}{|l|l|c|c|c|c|c|}
\hline & \multicolumn{2}{|c|}{ Clinicians' records } & \multicolumn{2}{c|}{ Patients' records } \\
\hline & $\begin{array}{c}\text { Previous } \\
\text { regimen }\end{array}$ & $\begin{array}{c}\text { rVIII- } \\
\text { SingleChai } \\
n\end{array}$ & P-value & $\begin{array}{c}\text { Previous } \\
\text { regimen }\end{array}$ & $\begin{array}{c}\text { rVIII- } \\
\text { SingleCha } \\
\text { in }\end{array}$ & P-value \\
\hline
\end{tabular}

physical activity (around 50\% of them). Patients on a prophylaxis regimen were younger and more engaged in sport activities compared with patients treated on-demand.

\section{Clinical results with rVIII-SingleChain and switch analysis}

Among patients reported in detail by physicians and patients (cohorts B and C), 27 and 20 patients, respectively, were currently on regular prophylaxis with rVIII-SingleChain after switching from a prophylactic regimen with another drug. A switch analysis, to assess bleeding rate and schedule of the rVIII-SingleChain treatment as compared with the previous one, was performed on these patients. Prior to the switch, the majority of them (74\% of patients in the physicians' population and $80 \%$ in the patients' population) were on a $\geq 3$ weekly dosing regimen. After patients had switched to prophylaxis with rVIII-SingleChain, these proportions were reduced to $44 \%$ and 55\%, respectively (Fig. 2). As compared to the prior regimen, the proportion of patients able to be treated with a 2 weekly administration schedule increased from $26-56 \%$ in the reported population and from $20-45 \%$ in the patients' population. Mean weekly infusions was significantly reduced from 2.74 before to 2.44 after switching to rFVIII-SingleChain in the physicians' population $(p=0.013)$. A non-significant reduction, from 3.10 to 2.60 , was observed in the patients' population $(p=0.885)$; in this cohort, the high SD value and the small sample size may have hindered significance.

\begin{tabular}{|c|c|c|c|c|c|c|}
\hline & \multicolumn{3}{|c|}{ Clinicians' records } & \multicolumn{3}{|c|}{ Patients' records } \\
\hline & $\begin{array}{l}\text { Previous } \\
\text { regimen }\end{array}$ & $\begin{array}{l}\text { rVIII- } \\
\text { Single- } \\
\text { Chain }\end{array}$ & $\mathrm{p}$-value & $\begin{array}{l}\text { Previous } \\
\text { regimen }\end{array}$ & $\begin{array}{l}\text { rVIII- } \\
\text { Single- } \\
\text { Chain }\end{array}$ & p-value \\
\hline $\begin{array}{l}\text { Mean } \\
\text { (SD) }\end{array}$ & $\begin{array}{l}2.74 \\
\quad(0.447)\end{array}$ & $\begin{array}{l}2.44 \\
\quad(0.506)\end{array}$ & 0.013 & $\begin{array}{l}3.10 \\
\quad(1.071)\end{array}$ & $\begin{array}{l}2.60 \\
(0.598)\end{array}$ & 0.885 \\
\hline Median & 3.0 & 2.0 & & 3.0 & 3.0 & \\
\hline
\end{tabular}

The reduction in weekly infusions was obtained without a parallel increase in FVIII dosage: the mean annual dosage of rVIII-SingleChain was $4320 \mathrm{IU} / \mathrm{Kg}$ (median: $4320 \mathrm{IU} / \mathrm{Kg}$; range: $2215-6646 \mathrm{IU} / \mathrm{Kg}$ ) in the 27 patients included in the clinicians' detailed population versus $4740 \mathrm{IU} / \mathrm{Kg}$ (median: $4500 \mathrm{IU} / \mathrm{Kg}$; range: $2215-7200 \mathrm{IU} / \mathrm{Kg}$ ) with the previous drug $(\mathrm{p}=0.178)$. The corresponding mean dosage per single infusion were $37 \mathrm{IU} / \mathrm{Kg}$ (median: $38 \mathrm{IU} / \mathrm{Kg}$ ) versus $36 \mathrm{IU} /$ $\mathrm{Kg}$ (median: $35 \mathrm{IU} / \mathrm{Kg})(\mathrm{p}=0.792)$. After switching to rVIIISingleChain, thus, HA patients received a lower number of FVIII administrations without significant changes in single dosages, with a resulting reduced annual consumption of FVIII.

The bleeding rate of rVIII-SingleChain on prophylaxis also significantly improved as compared with the previous treatment in the physicians' and patients' records, as shown in Table 3. The mean ABR in the last 12 months of treatment with rVIII-SingleChain was less than 1 in both the clinicians' and patients' reported populations, versus more than 2 during the last 12 months with the previous prophylaxis regimen (mean 0.96 vs. 2.15 and 0.71 vs. 2.46 events/year; $p=0.031$ and $p=0.018$, respectively). The percentage of patients with zero bleeds during one year of 
Table 3 Bleeding rates before and after treatment with rVIII-SingleChain (no. total and joint bleeds in the last 12 months of treatment with the previous drug and in the last 12 months of treatment with rVIII-SingleChain)

\begin{tabular}{|c|c|c|c|c|c|c|}
\hline & \multicolumn{6}{|l|}{ Number (\%) } \\
\hline & \multicolumn{3}{|c|}{$\begin{array}{l}\text { Clinicians' point of view (cohort B) } \\
(\mathrm{n}=27)\end{array}$} & \multicolumn{3}{|c|}{$\begin{array}{l}\text { Patients' point of view (cohort } \mathrm{C} \text { ) } \\
(\mathrm{n}=20)\end{array}$} \\
\hline & Previous treatment & rVIII-SingleChain & p-value & Previous treatment & rVIII-SingleChain & p-value \\
\hline \multicolumn{7}{|l|}{ Total bleeds/year } \\
\hline Can't recall & $7(26)$ & $4(15)$ & $\mathrm{p}=0.031$ & $7(35)$ & $3(15)$ & $\mathrm{p}=0.018$ \\
\hline$>3$ & $4(15)$ & $2(7)$ & & $3(15)$ & $0(0)$ & \\
\hline $1-3$ & $9(33)$ & $7(26)$ & & $7(35)$ & $9(45)$ & \\
\hline 0 & $7(26)$ & $14(52)$ & & $3(15)$ & $8(40)$ & \\
\hline Mean (SD) & $2.15(2.601)$ & $0,96(1.745)$ & & $2.46(2.727)$ & $0.71(0.849)$ & \\
\hline Range & $0.0,8.0$ & $0.0,6.0$ & & $0.0,10.0$ & $0.0,3.0$ & \\
\hline Median (IQR) & $1.0(3)$ & $0.0(1)$ & & $2.0(2)$ & $1.0(1)$ & \\
\hline \multicolumn{7}{|l|}{ Joint bleeds/year } \\
\hline Can't recall & $7(26)$ & $4(15)$ & $\mathrm{p}=0.039$ & $10(50)$ & $3(15)$ & $\mathrm{p}=0.013$ \\
\hline$>3$ & $4(15)$ & $2(7)$ & & $2(10)$ & $0(0)$ & \\
\hline $1-3$ & $6(22)$ & $7(26)$ & & $5(25)$ & $5(25)$ & \\
\hline 0 & $10(37)$ & $14(52)$ & & $3(15)$ & $12(60)$ & \\
\hline Mean & $1.70(2.430)$ & $0.87(1.604)$ & & $1.90(2.079)$ & $0.41(0.712)$ & \\
\hline Range & $0.0,8.0$ & $0.0,6.0$ & & $0.0,6.0$ & $0.0,2.0$ & \\
\hline Median (IQR) & $0.5(3)$ & $0.0(1)$ & & $1.5(2)$ & $0.0(1)$ & \\
\hline
\end{tabular}

rVIII-SingleChain treatment was $52 \%$ and $40 \%$, respectively, in the doctors' and patients' detailed populations, compared to $26 \%$ and $15 \%$ with the previous drug. Similar trends were observed for joint bleedings: $52 \%$ and $60 \%$ of patients reported zero joint bleeds after 12 months of rVIIISingleChain prophylaxis in the doctor's and patients' populations, respectively, versus $37 \%$ and $15 \%$ with the previous treatment. The mean AJBR significantly lowered from 1.70 to $0.87(\mathrm{p}=0.039)$ in the physicians' cohort and from 1.90 to $0.41(\mathrm{p}=0.013)$ in the patients' cohort.

\section{Satisfaction with rVIII-SingleChain: the clinicians' point of view}

According to clinicians' responses after one year of observation, the most convincing features of rVIII-SingleChain were the longer half-life as compared to traditional $\mathrm{rFVIII}$ $(36 \%)$, the efficacy (29\%) and the safety (21\%), albeit some of them pointed out the need for longer follow-ups to drive definitive conclusions. The need for a conversion factor for measuring FVIII levels (29\%) and the issues associated to laboratory monitoring and dosages $(14 \%)$ were reported as the less convincing features. $36 \%$ of interviewed doctors did not find any drawbacks with the use of rVIII-SingleChain.

In general, mean satisfaction ratings were quite high among physicians: 4.14 after one year, with $86 \%$ of clinicians affirming to be satisfied or very satisfied (57\% and $29 \%$, respectively) of their experience with rVIII-SingleChain, whereas $14 \%$ were neither satisfied nor unsatisfied. The main reasons for clinicians' satisfaction about their patients in prophylaxis with rVIII-SingleChain were: excellent responses/efficacy (25\%), bleeding reduction/no bleeding (22\%), greater compliance (19\%), safety (16\%), and fewer infusions (13\%).

\section{Satisfaction with rVIII-SingleChain: the patients' point of view}

Overall, patients' satisfaction was comparable to that reported by clinicians: $86 \%$ of them declared to be satisfied/very satisfied (54\% and 32\%, respectively) and $14 \%$ to be neither satisfied nor unsatisfied. Mean satisfaction rating was 4.18 . Notably, none of them affirmed to be not at all satisfied. Main satisfaction reasons reported by the patients were linked to both emotional wellbeing aspects ("I feel more protected", $41 \%$; "I'm calm/I feel better", 32\%) and to the drug's efficacy features ("I have reduced my number of infusions", $32 \%$; "positive continuity with the previous therapy", $18 \%$ ). $68 \%$ of patients did not find any area of dissatisfaction, whereas $32 \%$ of them mentioned areas of lower satisfaction mainly linked to the general burden of the disease (need for intravenous infusions, everyday life problems, and difficulties with the injections).

The last part of patients' interview was dedicated to explore their general experience with haemophilia and the 
Fig. 3 Impact of haemophilia on everyday life from the patients' perspective

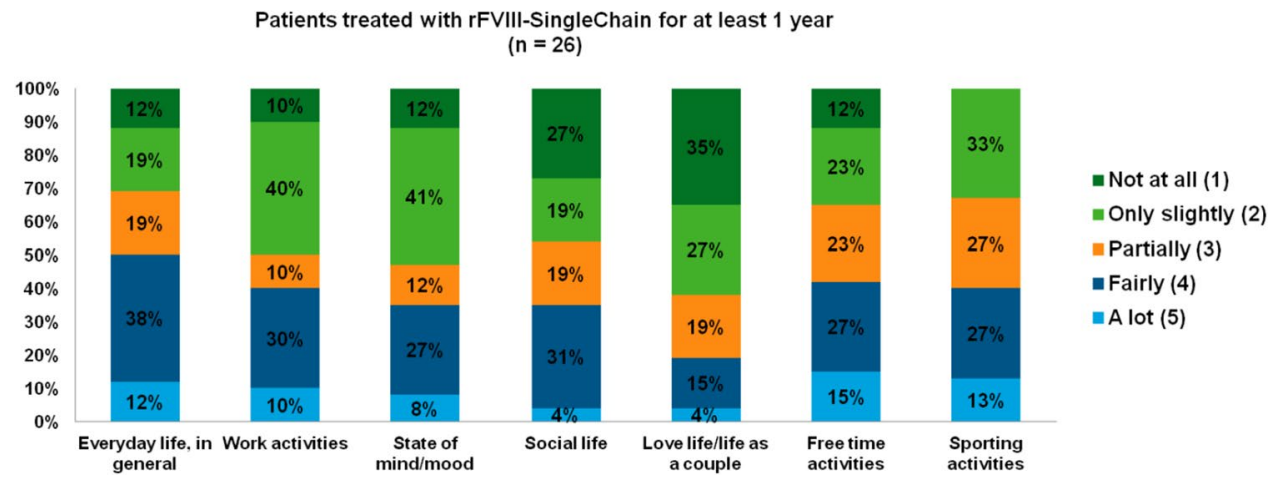

level of information on the disease. Required to describe their feelings about haemophilia, $35 \%$ of patients judged their daily life normal, and $23 \%$ to have to pay more attention, while $23 \%$ felt limited in life and $23 \%$ reported feelings of fear, anxiety, worry, and depression. Although prophylactic therapy was judged to confer more protection in everyday life and sport (45\% of patients) and higher freedom (30\%), $50 \%$ of patients still complained about the dependency on continuous infusions. Patients were requested to indicate how much illness interfered with their everyday life and with specific aspects of their life: the results are illustrated in Fig. 3. Among 26 patients treated with rVIII-SingleChain for at least 1 year (cohort $\mathrm{C}$ ), free time and sporting activities were the aspects of life most affected by the disease, whereas globally half of them $(50 \%)$ reported no or partial impact on everyday life, in general.

Overall, all the patients declared to have a good level of information on their disease and its treatments (85\% judged themselves fairly informed, and $15 \%$ very informed), mainly acquired by their treating physician (92\%). Other sources of information were websites/internet forums (58\%) and patients' associations (54\%). A good degree of satisfaction was reported by patients with the services offered by their HTC (mainly availability of medical staff in case of emergency, relationship with the doctor, and time dedicated to the patients), but expectations for improvements in services were still present: the most interesting services, not offered by the Centre, were the home delivery of drugs (46\%) and an office to handle bureaucratic matters (27\%).

\section{Discussion}

Our survey explored physicians' and patients' points of view and clinical experience after switching to rVIII-SingleChain for the treatment of HA. The results reinforced the good clinical performances of rVIII-SingleChain as previously reported in the pivotal clinical trials, namely the significant proportion of patients able to follow a twice per week prophylaxis regimen, without an increase in annual total FVIII consumption, and reduced ABRs and AJBRs. Moreover, the satisfaction ratings with rVIII-SingleChain were particularly elevated, highlighting treatment's efficacy and safety among the doctors and the positive impact on everyday life and emotional wellbeing among the patients.

The population described in our analysis included prevalently young patients with HA (mean age of about 30 years) actively engaged in physical activity, $73 \%$ of whom had a severe form of the disease and were treated on prophylaxis ( $94 \%$ of severe patients). Prophylaxis is recommended in patients with severe HA by current guidelines [10, 11], but limited data are available in Europe about its actual prescription in the clinical management of haemophilia patients [12]. The data obtained by our national sample suggest that prophylaxis is in fact the preferred treatment regimen in young severe HA patients in the Italian everyday clinical practice. 98\% population enrolled in this survey had received previous treatment before switching to rVIII-single chain therapy. Among the enrolled patients, about $60 \%$ underwent previous treatment, no longer available on the market, therefore clinicians opted for rVIIISingle Chain for its efficacy and safety features.

Compared to the previous treatment, a significant reduction in the number of weekly infusions was reported in patients receiving prophylaxis with rVIII-SingleChain: $56 \%$ of patients were able to follow a twice a week injection regimen as compared to $26 \%$ with the previous drug (clinicians' detailed population). Burden of care is a highly relevant factor associated with prophylaxis treatment in haemophilia patients; our data suggest that in a clinical practice setting, more than half of HA patients treated with rFVIIISingleChain, including the majority of patients with severe disease, can be safely and effectively dosed with a twice a week prophylactic regimen, with an overall reduction of treatment burden as compared to other full length rFVIII products.

In the face of a reduced number of injections, no increases in FVIII consumption was observed; in fact a nonsignificant reduction in the patient's mean annual dosage was reported in the switch analysis of prophylaxis patients. 
Notwithstanding the lack of statistical significance, likely due to the small sample size as a major factor, the net numerical difference (29.400 IU/year for a patient with a body weight of $70 \mathrm{~kg}$ ) may have very relevant economic implications for national health care systems when costs associated with haemophilia care are considered. These data are especially important since a higher consumption and expenditure have been reported in patients who had switched from an SHL to an EHL product in a real-world analysis (not including rVIII-SingleChain) [13], and confirm the improved PK parameters showed by rVIII-SingleChain as compared to classical SHL products [5]. It must be noted that the majority (66\%) of the 35 patients currently on prophylaxis with rVIIISingleChain and previously treated with prophylaxis used 2nd generation recombinant FVIII and none of them used EHL products. Although rVIII-SingleChain was not strictly classified as a long-acting rFVIII product [14], improved PK parameters have been demonstrated versus standard half-life products (i.e. octocog alfa) $[5,15]$. Our findings confirm the clinical relevance of these observations and suggest a longer half-life of rVIII-SingleChain compared to classical SHL products in the clinical setting.

Low ABRs were reported by clinicians and patients treated with rVIII-SingleChain in our survey, with a mean of 0.96-0.71 (respectively) events observed in 1 year of treatment. By comparison, the mean ABRs reported by doctors and patients with the previous drug were 2.15 and 2.46, suggesting an improved bleeding control associated to rVIII-SingleChain prophylaxis. Similar data were observed in relation to joint bleedings, whose annual rates showed a decrease as compared with the previous treatment in the physicians' and patients' reports. Overall, 52-60\% of prophylaxis patients (clinicians' and patients' cohorts, respectively) did not reported any joint bleeds after one year of rVIII-SingleChain treatment, as compared to 37\% and $15 \%$, respectively, with the previous products. Prevention of arthropathy and improvement of QoL are the main goals of prophylaxis in severe HA patients: in the pivotal rVIII-SingleChain trials median overall ABRs of 1.14 and 3.69 were observed in severe adult and children patients, respectively $[7,8]$. The low bleeding rates reported in our clinical practice survey demonstrate that an efficient control of bleeding events can be obtained with rVIII-SingleChain in association with a simplified prophylactic regimen and reduced infusion frequency in a real-world setting.

Reduction in weekly infusions and high haemostatic efficacy in patients receiving prophylaxis with rVIII-SingleChain have recently been confirmed also in two European and American real-world studies. Olivieri et al. demonstrated that in a population of 225 male HA German patients, after switching to rFVIII-SingleChain prophylaxis, the mean FVIII consumption was reduced by $32 \%$ compared with prior FVIII products and the percentage of patients receiving $\leq 2$ infusions/week increased from 0 to $71.4 \%$. Excellent bleeding protection was reported, with similar or potentially lower bleeding rates than standard-acting FVIII therapies and a proportion of patients with zero spontaneous bleeds after switching to prophylaxis with rFVIII-SingleChain improved from 76.2-95.2\% [9]. Similar data were reported in a real-world analysis of 120 male US patients receiving prophylaxis with three different EHL FVIII products, including rFVIII-SingleChain, rFVIIIFc, or PEG-rFVIII: prophylaxis with rFVIII-SingleChain presented lower mean factor consumption than both the other two FVIII products $(-11 \%$ and $-13.7 \%$, respectively), with comparable bleed rates [6]. Our data support these findings.

Real-world studies are useful to validate clinical trials' results in the daily clinical practice, a setting displaying different issues and problems. Moreover, they allow insights on personal experience, level of satisfaction and critical areas amid health workers and patients under everyday conditions. The double perspective, the medical point of view of the clinicians and the experiential perspective of patients who have to daily manage this disease, was a distinct feature of our work. Prophylactic treatment of haemophilia, although effective, imposes a significant burden of care on patients' everyday life. Patients' distress and the presence of anxiety and depression are key factors in the relationship of haemophilia patients with their disease and may influence adherence to replacement therapy [16, 17], shaping a point of view on the illness potentially different from that of the specialists involved in their care. Notably, although the two detailed populations analysed in our study were not completely superimposable, and thus not directly comparable, patients' reports appeared substantially in accord with the vision of their physicians. Both doctors and patients, when requested to compare rVIII-SingleChain prophylaxis with the previous treatment, identified the number of bleeds and the reduction in injection frequency as areas of improvement. Satisfaction rates were also comparable in the two populations, reaching more of $85 \%$ of satisfied/very satisfied among both clinicians and patients. However, some distinction between doctors' and patients' points of view became evident in the analysis of the main reasons of satisfaction. Whereas clinicians mainly underscored efficacy/ safety features of rVIII-SingleChain, patients prevalently highlighted the positive effect on their QoL and emotional wellbeing. Patients reported a reduction in the impact of haemophilia on their work life, their state of mind and mood, and on their general everyday life. Positive expression such as "protection" and "peace-of-mind" could be found among patients' spontaneous comments regarding their experiences with haemophilia. The relevance of these findings may be significant in the clinical practice, since "patients' acceptance" and "compliance to therapy" were described within the major barriers to prophylaxis in haemophilia patients. On 
the contrary, "bleeding frequency" and "presence of target joints" were factors favouring the prescription of prophylaxis among clinicians [12]. Overall, doctors' and patients' perceptions may remain different, because different are the main focuses and expectations (an effective and safe treatment versus a good $\mathrm{QoL}$ and perception of being protected against the disease). These considerations underline the value of the matching assessment and satisfaction on rVIII-SingleChain reported by clinicians and patients in the present survey, suggesting that the drug is able to favourably conjugate different points of view on haemophilia prophylactic treatment. Reducing the burden associated with prophylaxis while maintaining a high haemostatic protection without FVIII increased consumption might significantly improve clinical outcomes and QoL in haemophilia patients.

The main limitations of the current survey are related to the small sample size and to the limits of the tool itself. Although representative of the entire national condition, our specialist sample may suffer of potential selection bias. At the same time, since the participation of patients was voluntary, we were not able to completely match the clinicians' and patients' reported populations. Although a limit, this issue might also allow for an independent comparison between two different points of view, which, as already reported, appeared to be strikingly similar and complementary in their dissimilarities. In the interpretation of the results, surveys, as every self-reported outcome, may suffer of a lack of objectivity. On the other side, they offer a unique opportunity to collect the point of view of all the actors involved. We are well aware that our findings cannot substitute an observational study, but also believe that self-reported data have a value specifically related to their subjectivity. While acknowledging the limitation of this study, the results presented here show that both clinicians and patients felt that switching to rVIII-SingleChain reduced treatment burden and bleeding events, increasing their satisfaction with prophylactic haemophilia treatment, a finding worth to be kept in mind in the choice of therapeutic options for this disease.

In conclusion, this study evaluated the benefits associated with rVIII-SingleChain treatment in a cohort of specialists and patients from HTCs in Italy.

Results of this real-world study for rVIII-SingleChain support data reported in the clinical studies [18]. The survey results highlight the clinicians' indication of rVIIISingle Chain as a reliable choice with a favorable pharmacokinetic profile. Responses to the survey were based on the treatment efficacy (evaluated as bleeding reduction or absence of bleeding), safety, and favorable half-life.

The analysis of their clinical experience suggests that switching to rVIII-SingleChain allowed patients to reduce their dosing frequency without increasing factor consumption or compromising clinical outcome. Both clinicians and patients reported high levels of satisfaction with the treatment, pointing out its efficacy and positive impact on everyday life and emotional wellbeing.

GF, AF, PG, ML, GM, MM, ACM, BP, MS and EZ declare that they have no commercial association(e.g. consultancies, stock ownership, equity interest, patent/licensing arrangements etc.) that might pose a conflict of interest in connection with the submitted article.

Acknowledgements The Authors thanks IQVIA ${ }^{\mathrm{TM}}$ that has carried out the present survey and performed all the statistical data analyses. The authors thanks also Enrica Orsini on behalf of Mattioli 1885 for editorial assistance.

Funding The survey and the medical writing assistance service were sponsored by CSL Behring, Italy, which did not influence the results output/intervene in the definition of the results.

\section{Declarations}

Conflict of interest $\mathrm{AB}$ received honoraria for speaker's bureau and/ or participation in Advisory Boards from Bayer, CSL Behring, Kedrion, Novo Nordisk, Roche, Sobi, Takeda. GC received honoraria for speaker's bureau and/or participation in Advisory Boards for Alexion. Bayer, Biomarin, CSL Behring, Grifols, Kedrion, LFB, NovoNordisk, Roche, Sanofi, Sobi, Takeda, Uniqure, Werfen. AR acted as paid consultant/member of Advisory Board/speaker for Bayer, CSL Behring, Kedrion, NovoNordisk, Roche, Sobi, Takeda. CS received honoraria for speaker's bureau and/or participation in Advisory Boards from Bayer, Takeda, CSL Behring, Sobi, Roche, NovoNordisk.

Open Access This article is licensed under a Creative Commons Attribution 4.0 International License, which permits use, sharing, adaptation, distribution and reproduction in any medium or format, as long as you give appropriate credit to the original author(s) and the source, provide a link to the Creative Commons licence, and indicate if changes were made. The images or other third party material in this article are included in the article's Creative Commons licence, unless indicated otherwise in a credit line to the material. If material is not included in the article's Creative Commons licence and your intended use is not permitted by statutory regulation or exceeds the permitted use, you will need to obtain permission directly from the copyright holder. To view a copy of this licence, visit http://creativecommons.org/licenses/by/4.0/.

\section{References}

1. Mannucci PM, Tuddenham EG (2001) The hemophilias-from royal genes to gene therapy. N Engl J Med 344(23):1773-1779

2. Oldenburg J (2015) Optimal treatment strategies for hemophilia: achievements and limitations of current prophylactic regimens. Blood 125(13):2038-2044

3. Manco-Johnson MJ, Abshire TC, Shapiro AD et al (2007) Prophylaxis versus episodic treatment to prevent joint disease in boys with severe hemophilia. N Engl J Med 357(6):535-544

4. Al-Salama ZT, Scott LJ (2017) Lonoctocog alfa: a review in haemophilia A. Drugs 77(15):1677-1686

5. Klamroth R, Simpson M, von Depka-Prondzinski M et al (2016) Comparative pharmacokinetics of rVIII-singlechain and 
octocog alfa (Advate $((\mathrm{R})))$ in patients with severe haemophilia A. Haemophilia 22(5):730-738

6. Simpson ML, Desai V, Maro GS, Yan S (2020) Comparing factor use and bleed rates in U.S. hemophilia A patients receiving prophylaxis with 3 different long-acting recombinant factor VIII products. J Manag Care Spec Pharm 26(4):504-512

7. Mahlangu J, Kuliczkowski K, Karim FA et al (2016) Efficacy and safety of rVIII-singlechain: results of a phase $1 / 3$ multicenter clinical trial in severe hemophilia A. Blood 128(5):630-637

8. Stasyshyn O, Djambas Khayat C, Iosava G et al (2017) Safety, efficacy and pharmacokinetics of rVIII-singlechain in children with severe hemophilia A: results of a multicenter clinical trial. J Thromb Haemost 15(4):636-644

9. Olivieri M, Sommerer P, Maro G, Yan S (2020) Assessing prophylactic use and clinical outcomes in hemophilia A patients treated with rVIII-singlechain and other common rFVIII products in Germany. Eur J Haematol 104(4):310-317

10. Srivastava A, Brewer AK, Mauser-Bunschoten EP et al (2013) Guidelines for the management of hemophilia. Hemophilia 19(1): $1-\mathrm{e} 47$

11. Richards M, Williams M, Chalmers E et al (2010) A United Kingdom haemophilia centre doctors' organization guideline approved by the British committee for standards in haematology: guideline on the use of prophylactic factor VIII concentrate in children and adults with severe haemophilia A. Br J Haematol 149(4):498-507

12. Castaman G, Rocino A, Mazzucconi MG, Zanon E, Gagliano F, Molinari AC (2015) Prophylaxis therapy in paediatric patients with haemophilia: a survey of clinical management trends in Italy. Blood Transfus = Trasfusione del sangue 13(4):631-638

13. Chhabra A, Fogarty PF, Tortella BJ et al (2018) Real-world analysis of dispensed international units of coagulation factor VIII and resultant expenditures for hemophilia A patients: a comparison between standard half-life and extended half-life products. Manag Care 27(10):39-50

14. Mahlangu J, Young G, Hermans C, Blanchette V, Berntorp E, Santagostino E (2018) Defining extended half-life rFVIII-a critical review of the evidence. Haemophilia 24(3):348-358

15. Graf L (2018) Extendedhalf-life factor VIII and factor IX preparations. Transfus Med Hemother 45(2):86-91

16. Tran DQ, Barry V, Antun A, Ribeiro M, Stein S, Kempton CL (2017) Physician trust and depression influence adherence to factor replacement: a single-centre cross-sectional study. Haemophilia 23(1):98-104

17. Pinto PR, Paredes AC, Moreira P et al (2018) Emotional distress in haemophilia: factors associated with the presence of anxiety and depression symptoms among adults. Haemophilia 24(5):e344-e353

18. Mahlangu J, Kuliczkowski K, Karim FA et al (2016) Efficacy and safety of rVIII-SingleChain: results of a phase 1/3 multicenter clinical trial in severe hemophilia A. Blood 128(5):630-637. https://doi.org/10.1182/blood-2016-01-687434

Publisher's Note Springer Nature remains neutral with regard to jurisdictional claims in published maps and institutional affiliations.

\section{Authors and Affiliations}

\section{Alessandra Borchiellini ${ }^{1}$ (1) - Giancarlo Castaman ${ }^{2} \cdot$ Giulio Feola $^{3} \cdot$ Antonietta Ferretti $^{4} \cdot$ Paola Giordano $^{5}$. Matteo Luciani ${ }^{6}$. Giuseppe Malcangi ${ }^{7}$. Maurizio Margaglione ${ }^{8}$. Angelo Claudio Molinari ${ }^{9} \cdot$ Berardino Pollio $^{10}$. Angiola Rocino ${ }^{11} \cdot$ Cristina Santoro $^{12} \cdot$ Michele Schiavulli $^{13} \cdot$ Ezio Zanon $^{14}$}

Giancarlo Castaman

castaman@aou-careggi.toscana.it

Giulio Feola

gi.feola@aslsalerno.it

Antonietta Ferretti

ferretti@bce.uniroma1.it

Paola Giordano

paola.giordano@uniba.it

Matteo Luciani

matteo.luciani@opbg.net

Giuseppe Malcangi

giuseppe.malcangi@policlinico.ba.it

Maurizio Margaglione

m.margaglione@unifg.it

Angelo Claudio Molinari

aclaudiomolinari@gaslini.org

Berardino Pollio

bpollio@cittadellasalute.to.it

Angiola Rocino

anrocino@gmail.com

Cristina Santoro

santoro@bce.uniroma1.it
Michele Schiavulli

mischiavulli@gmail.com

Ezio Zanon

ezio.zanon@unipd.it

1 Centro di Riferimento Regionale Malattie Emorragiche e Trombotiche dell'adulto Ematologia U Città della Salute, Torino, Italy

2 Department of Oncology, Center for Bleeding Disorders and Coagulation, Careggi University Hospital, Florence, Italy

3 Centro Emofilia di Vallo della Lucania, Salerno, Italy

4 Department of Translational and Precision Medicine, Sapienza University, Rome, Italy

5 Paediatric Section, Department of Biomedicine and Human Oncology, University of Bari, Bari, Italy

6 Oncohematology Department Bambino, Gesù Pediatric Hospital, Rome, Italy

7 UOSD Emofilia e Trombosi Azienda Ospedaliero Universitaria Policlinico di Bari, Bari, Italy

8 Genetica Medica Dip.to Medicina Clinica e Sperimentale Università di Foggia, Foggia, Italy

9 Regional Reference Center for Hemorrhagic Diseases, Giannina Gaslini Children's Hospital, Genoa, Italy 
10 Centro di Riferimento Regionale Malattie Emorragiche e Trombotiche Ereditarie in età pediatrica, S.S.D. Medicina Trasfusionale Materno-Infantile-Traumatologica, Azienda Ospedaliera Citta' Della Salute e della Scienza-Ospedale Infantile Regina Margherita, Turin, Italy

11 Hematology Unit-Haemophilia and Thrombosis Centre, Ospedale del Mare, Napoli, Italy

12 Hematology, University Hospital Policlinico Umberto I, Rome, Italy
13 Dipartimento di Oncologia, Centro di Riferimento Regionale per le Emocoagulopatie, AORN Santobono Pausilipon, Napoli, Italy

14 Haemophilia Centre, Department of Medicine, University Hospital of Padua, Padua, Italy 